\title{
Modelling Of Automotive Fuel Droplet Heating and Evaporation: Recent Results and Unsolved Problems
}

\author{
S. S. Sazhin \\ The Sir Harry Ricardo Laboratories, Advanced Engineering Centre \\ School of Computing, Engineering and Mathematics \\ University of Brighton, UK
}

The most recent developments in the modelling of heating and evaporation of automotive fuel droplets, the results of which were published in 2014-2016, are reviewed, and the most important unsolved problems are identified. Several approaches to modelling the heating of evaporating droplets, predicting different heating and evaporation characteristics, are compared. New results in modelling heating and evaporation of spheroidal droplets are identified. Basic principles of the Discrete Component (DC) model and its application to biodiesel fuel droplets are summarised. Main ideas of the Multidimensional Quasi-discrete (MDQD) model and its applications to Diesel and gasoline fuel droplets are discussed. A selfconsistent kinetic model for droplet heating and evaporation is described. New approaches to the estimation of the evaporation coefficient, including those taking into account quantum-chemical effects, are summarised. Among unsolved problems, the effects of non-spherical droplets, limitations of the ETC/ED model, effects of the interaction between droplets, modelling of droplet heating and evaporation in near- and super-critical conditions, development of advanced kinetic and molecular dynamics models and effective approximation of the kinetic effects are discussed. 